Portland State University

PDXScholar

Physics Faculty Publications and Presentations

Physics

$1-1-2009$

\title{
Dark current behavior in DSLR cameras
}

Justin Charles Dunlap

Portland State University

Oleg Sostin

Ralf Widenhorn

Portland State University

Erik Bodegom

Portland State University

Follow this and additional works at: https://pdxscholar.library.pdx.edu/phy_fac

Part of the Physics Commons

Let us know how access to this document benefits you.

\section{Citation Details}

Justin C. Dunlap ; Oleg Sostin ; Ralf Widenhorn and Erik Bodegom, "Dark current behavior in DSLR cameras", Proc. SPIE 7249, Sensors, Cameras, and Systems for Industrial/Scientific Applications X, 72490N (January 27, 2009); http://dx.doi.org/10.1117/12.806128

This Article is brought to you for free and open access. It has been accepted for inclusion in Physics Faculty Publications and Presentations by an authorized administrator of PDXScholar. Please contact us if we can make this document more accessible: pdxscholar@pdx.edu. 


\title{
Dark current behavior in DSLR cameras
}

\author{
Justin C. Dunlap, Oleg Sostin, Ralf Widenhorn, and Erik Bodegom \\ Portland State, Portland, OR 97207
}

\begin{abstract}
Digital single-lens reflex (DSLR) cameras are examined and their dark current behavior is presented. We examine the influence of varying temperature, exposure time, and gain setting on dark current. Dark current behavior unique to sensors within such cameras is observed. In particular, heat is trapped within the camera body resulting in higher internal temperatures and an increase in dark current after successive images. We look at the possibility of correcting for the dark current, based on previous work done for scientific grade imagers, where hot pixels are used as indicators for the entire chip's dark current behavior. Standard methods of dark current correction are compared to computed dark frames. Dark current is a concern for DSLR cameras as optimum conditions for limiting dark current, such as cooling the imager, are not easily obtained in the typical use of such imagers.
\end{abstract}

Keywords: Dark current, image correction, digital imagers, DSLR, CCD, CMOS

\section{INTRODUCTION}

It has been shown in prior work ${ }^{1}$ that dark current, noise generated due to thermal excitation of electrons within a digital imager, can be globally corrected in a Charge-Coupled Device (CCD) by analyzing pixels that show the greatest amounts of dark current relative to other pixels on the imager. These pixels, referred to as hot pixels, are caused by impurities in the silicon of the pixel. ${ }^{2,3,4}$ This method has further been shown to work in Complimentary Metal-Oxide Semiconductor (CMOS) digital imagers. ${ }^{5}$ However, these studies have been carried out on scientific grade imagers where the temperature of the imager can be carefully controlled or in imagers outside of a camera body. These methods had not previously been shown to work for common consumer grade cameras, nor for environments easily obtainable by a typical camera user. Therefore, the scope of this work is to document the behavior of dark current in DSLR cameras and to analyze the possibility of using this method of correction in such cameras.

The measurement of dark current in DSLR cameras is obfuscated by inconsistent internal temperature within the camera body and by camera manufactures' own efforts to deal with dark noise. As dark current is one of the largest sources of noise in digital imagers, camera manufactures implement their own systems for removing the noise to provide better pictures at longer exposure times. Most commonly for images at long exposures, a second image with a closed shutter will be taken after the initial image. This second image is a dark frame, an image with no light signal that is subtracted from the initial image to remove the dark noise and will ideally leave only the light information. However, there are problems associated with this method of correction. Foremost, this method is time consuming as it is required after every exposure. Furthermore, the camera will not be under identical conditions for the second image due to the generation of the first image itself. As is shown in this paper, the dark current is not consistent from frame to frame and shows an increase after an increasing number of frames are taken in succession likely this is due to an increase in the internal temperature of the camera. In addition, due to the statistical nature of the dark current even dark frames taken under identical conditions will not necessarily be the same.

Two of the presented cameras, the Nikon D70 and Nikon D700, appear to have a median filter applied to the image after collection. A median filter removes values of pixels with inconsistently large values as compared with their neighbors by applying an average or median value of those neighboring pixels to the unusually high valued pixels whether the noise reduction mode is turned on or not. The noise reduction mode appears to deal with dark noise by taking a second image as described in the paragraph above. It was found that by enabling the noise reduction mode and turning the camera off before it was able to collect the second image, no median filter would be applied to the image and the dark current in the first image was saved. ${ }^{6}$

While camera manufactures do deal with the problem of dark noise in their cameras, they do not necessarily do the best possible job. In addition to studying the behavior of the dark current, we also attempt to improve on the methods used by the camera manufactures by using the method of analyzing the hot pixels as dark current indicators for the entire image.

Sensors, Cameras, and Systems for Industrial/Scientific Applications X,

edited by Erik Bodegom, Valérie Nguyen, Proc. of SPIE-IS\&T Electronic Imaging, SPIE Vol. 7249, 72490N

(C) 2009 SPIE-IS\&T · CCC code: 0277-786X/09/\$18 - doi: 10.1117/12.806128 


\section{DARK CURRENT BEHAVIOR}

We present data from four commercially available DSLR consumer cameras. Images were taken with a Canon EOS20D, Canon EOS-20Da, Nikon D70, and Nikon D700. All of the cameras generate a raw file format, an image that is uncompressed and that has one measurement value for every pixel on the imager. Raw images are necessary for dark current correction due to the loss of information involved in processed images. These cameras all generated 12 bit pixel values except for the Nikon D700 which has a 14 bit pixel depth. Images were taken in a temperature controlled, light sealed enclosure. The cameras were allowed to reach thermal equilibrium with the air inside the box prior to obtaining images and the temperature was monitored inside the enclosure throughout the image taking process.

\subsection{Canon EOS-20D}

The Canon 20D has a $22.5 \times 15.0 \mathrm{~mm}$ CMOS sensor, with 8.2 million effective pixels in a grid of 3522 by 2348 pixels. To initially gauge the behavior of dark current in the Canon 20D, we collected images at varying exposure times starting at $30 \mathrm{~s}$ descending to $5 \mathrm{~s}$. Ten frames were taken at each exposure time and an average was taken for each pixel. The camera was set to an ISO setting of 1600 and the external temperature within the enclosure was $24^{\circ} \mathrm{C}$ with a temperature increase within the enclosure of approximately a degree for the entire run. Figure 1a shows the response of eight pixels on the imager. Pixel coordinates are given in parentheses for each pixel number. Pixels 1 through 7 are hot pixels to varying degrees and Pixel 8 is an example of a typical pixel on the imager that appears to have little dark current. After $20 \mathrm{~s}$ there appears to be a flattening of counts with additional length in exposure time resulting in marginal change in dark counts for all pixels. As shown in Fig. 1b, when the exposures were taken such that the $5 \mathrm{~s}$ exposures were taken first and then by ascending value to $30 \mathrm{~s}$, the counts no longer flattened after $20 \mathrm{~s}$. Pixel 1 is saturated after $25 \mathrm{~s}$ at the maximum count and therefore does not show an increase in counts from 25 to $30 \mathrm{~s}$. An increase in internal temperature of the imager with each additional frame would result in more dark current in images taken later on in the run. This would affect the exposure time trend by apparently decreasing values for the exposure time taken first and could account for the trend seen in Fig. 1a.
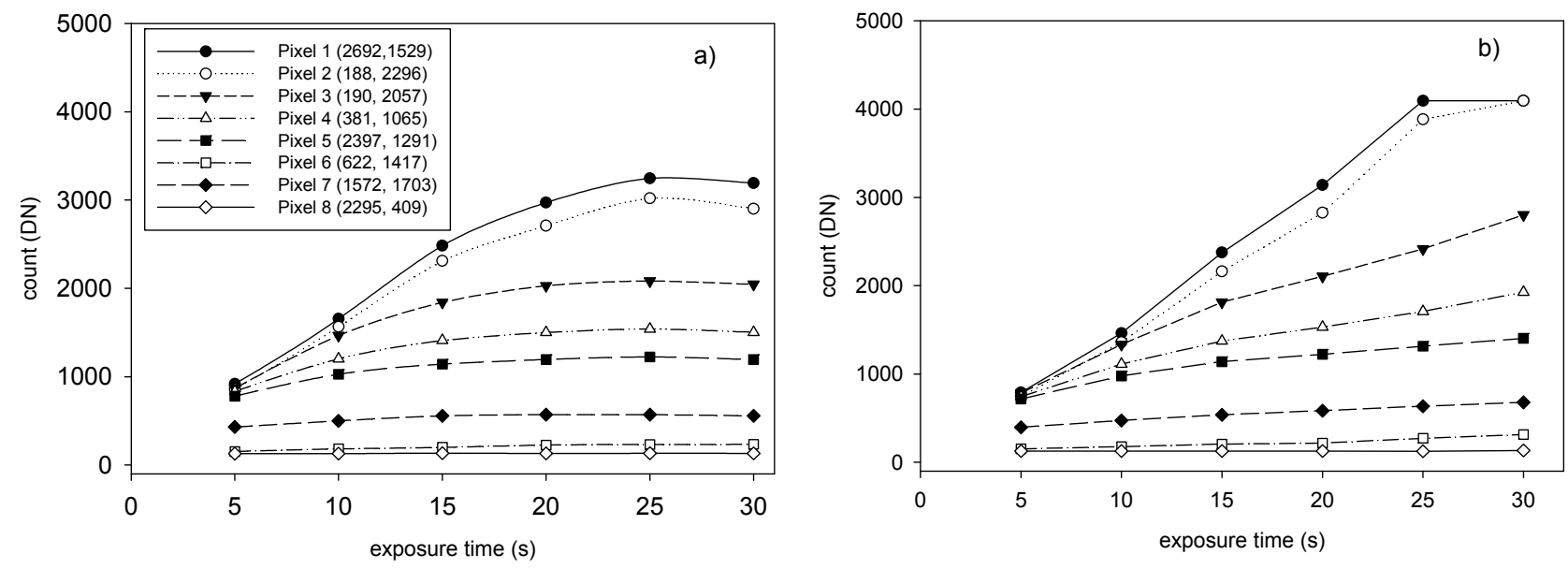

Fig. 1. Exposure time dependence of dark current for eight pixels, taken at $\mathrm{ISO}=1600$ and $24^{\circ} \mathrm{C}$. Panel a): $30 \mathrm{~s}$ images taken first descending to $5 \mathrm{~s}$ images. Panel b): $5 \mathrm{~s}$ images taken first ascending to $30 \mathrm{~s}$ images.

Shown in Fig. 2 are the counts for eight pixels in the $30 \mathrm{~s}$ frames used to find the average in Fig. 1a. For each pixel there is an increase in counts for each successive frame, with an increase of a third or more from the first frame to the last. This increase in value further lends evidence to the possibility that the internal temperature within the camera, and of the chip itself, increases significantly from each frame to the next. As discussed in the introduction, a common method for correcting for dark current is to take a dark frame immediately after the initial exposure. This method assumes similar conditions for the two frames, however from Fig. 2 it is evident that the act of obtaining a frame has changed the condition of the imager and the second frame will likely have more dark noise than the initial frame.

To further study the dependence of dark noise on preceding frames, we took 100 frames each with an integration time of $30 \mathrm{~s}$ with $5 \mathrm{~s}$ between frames. The temperature within the enclosure started at $24.0^{\circ} \mathrm{C}$ and increased to $25.2^{\circ} \mathrm{C}$ at the one hundredth frame. The hot pixels shown in Fig. 3 show a large increase in dark noise from the first frame to the last. The increase of $1.2^{\circ} \mathrm{C}$ in external temperature is not large enough to account for such changes in dark noise. This again 
shows that the internal temperature of the camera and the temperature of the sensor itself likely increases more dramatically than the external temperature. In a series of multiple frames with a rest period between sets of ten, it appeared, for this specific camera, to take a rest period of two to three minutes for the pixel values to return to where they started in the first frame.

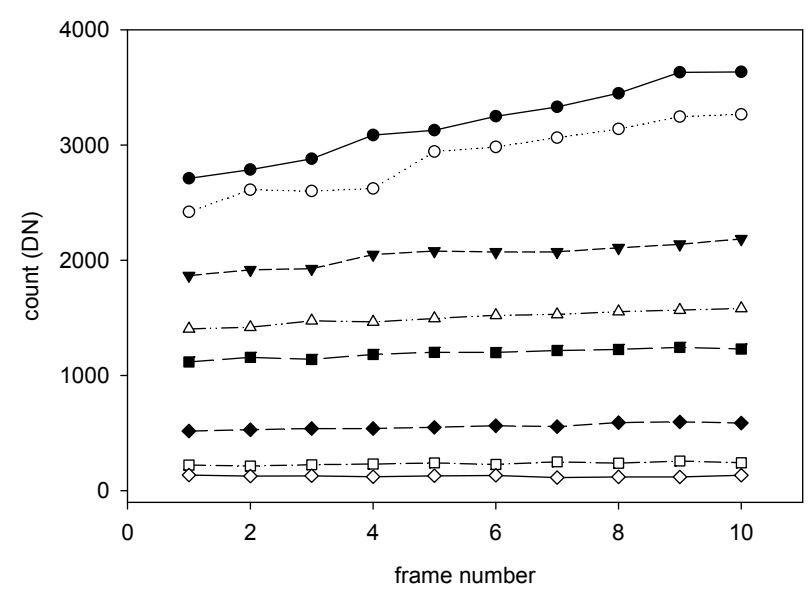

Fig. 2. Dark current dependence on frame number of eight hot pixels for ten $30 \mathrm{~s}$ frames separated by $5 \mathrm{~s}$ between frames, taken at ISO $=1600$ and $24^{\circ} \mathrm{C}$. (Same pixels shown as in Fig. 1.)

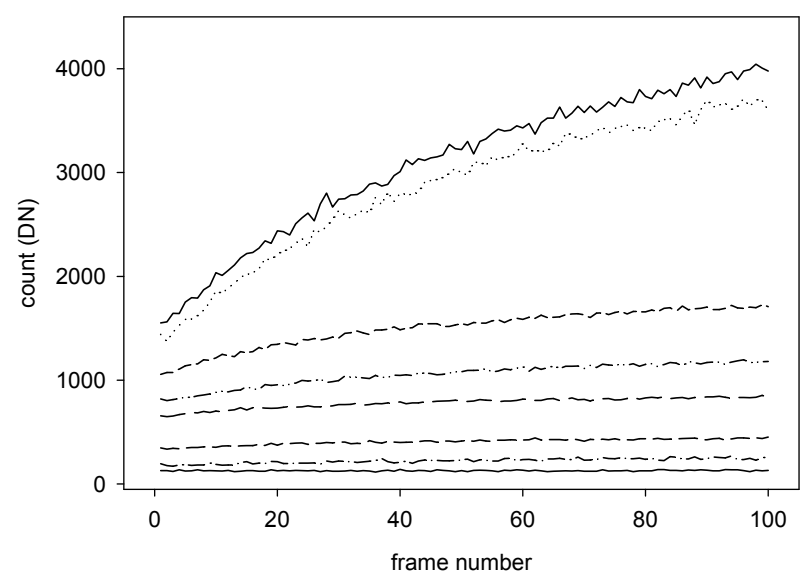

Fig. 3. Dependence of dark current for eight hot pixels on frame number for 100 frames with an integration time of $30 \mathrm{~s}$ and a rest period of $5 \mathrm{~s}$ between frames at $\mathrm{ISO}=1600$. (Same pixels shown as in Fig. 1.)

A series of exposures were taken in an attempt to more accurately determine the pixels' response to temperature. The Canon 20D was placed within the enclosure with an adjustable heater. The heater was adjusted to maintain a steady rise in temperature over the span of 105 minutes from a starting temperature of $24.8^{\circ} \mathrm{C}$ to $41.3^{\circ} \mathrm{C} .20 \mathrm{~s}$ exposures were taken every five minutes at an ISO setting of 800. Shown in Fig. 4 is the response of the eight pixels for this series. As is evident from the temperature response of the pixels shown in Fig. 4, the extreme increase in dark counts shown in Fig. 3 cannot be explained by change in external temperature alone. In addition, Fig. 4 highlights the possibility of using the pixels most responsive to temperature changes as dark current indicators. The pixel responses are predictable for temperature and exposure time and the hottest pixels, pixels with the greatest dark current signal to noise ratio, can be used to predict and correct the dark count of other pixels on the imager.

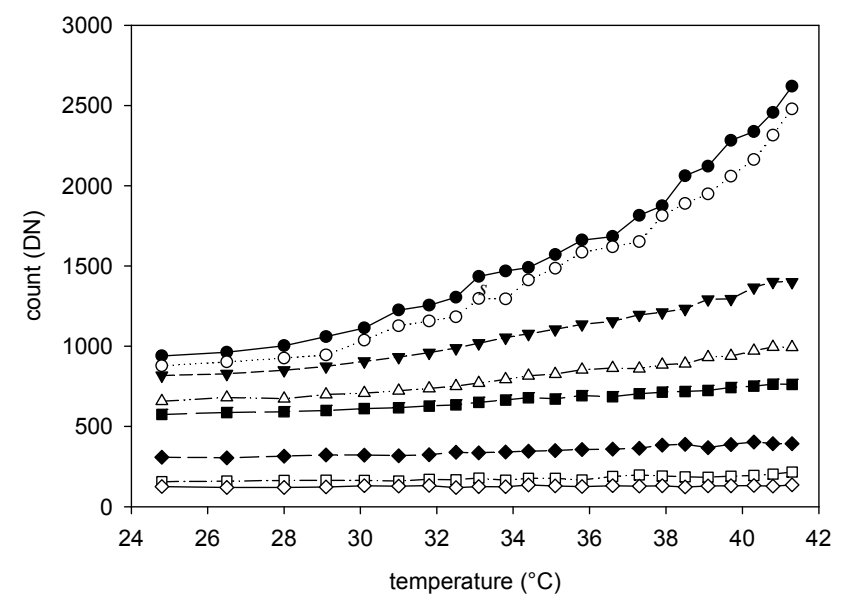

Fig. 4. Dependence of dark current for eight pixels on external temperature. $20 \mathrm{~s}$ exposures taken with an ISO setting of 800. (Same pixels shown as in Fig. 1.) 


\subsection{Canon EOS-20Da}

While the Canon 20Da and Canon 20D are very similar cameras, Canon modified the Canon 20Da from the Canon 20D for astronomical imaging to increase the sensitivity at the $\mathrm{H}$-alpha line and allow for real-time focusing on the display. A study of the Canon 20Da further demonstrates the predictable nature of pixels on similar imagers and shows that the Canon 20D is not an anomaly. Like the Canon 20D, the Canon 20Da has a $22.5 \times 15.0 \mathrm{~mm}$ CMOS sensor, with 8.2 million effective pixels in a grid of 3522 by 2348 pixels. Ten pictures were taken at exposure times of 5, 10, 15, 20, 25, and $30 \mathrm{~s}$ at a temperature of $24^{\circ} \mathrm{C}$ and at an ISO setting of 800 . Figure 5a shows average pixel values for eight pixels on the imager and their response to variation in exposure time. To study the effect of ISO setting on dark current, ten pictures were taken at ISO settings of $100,200,400,800$, and 1600 at a temperature of $24^{\circ} \mathrm{C}$ and with an exposure time of $30 \mathrm{~s}$. Figure 5b, shows the average pixel values of the same pixels as in Fig. $5 \mathrm{a}$ and their dependence on ISO setting. Pixel 8 is representative of most pixels on the imager and shows essentially no change in value for increasing exposure time. Pixels 1 to 7 represent pixels that have a large increase in values with increasing exposure time, as well as ISO setting, and therefore are also good candidates for indicating the dark current on this imager. However, Pixels 1 and 2 begin to saturate after an ISO setting of 400 and are therefore not good indicator pixels for this range. The pixels show a linear response with ISO setting as a change in ISO setting is essentially a change in the gain setting. As these pixels do show predictable behaviors with varying ISO setting, it is another factor that should intrinsically be taken into account when correcting for dark current.
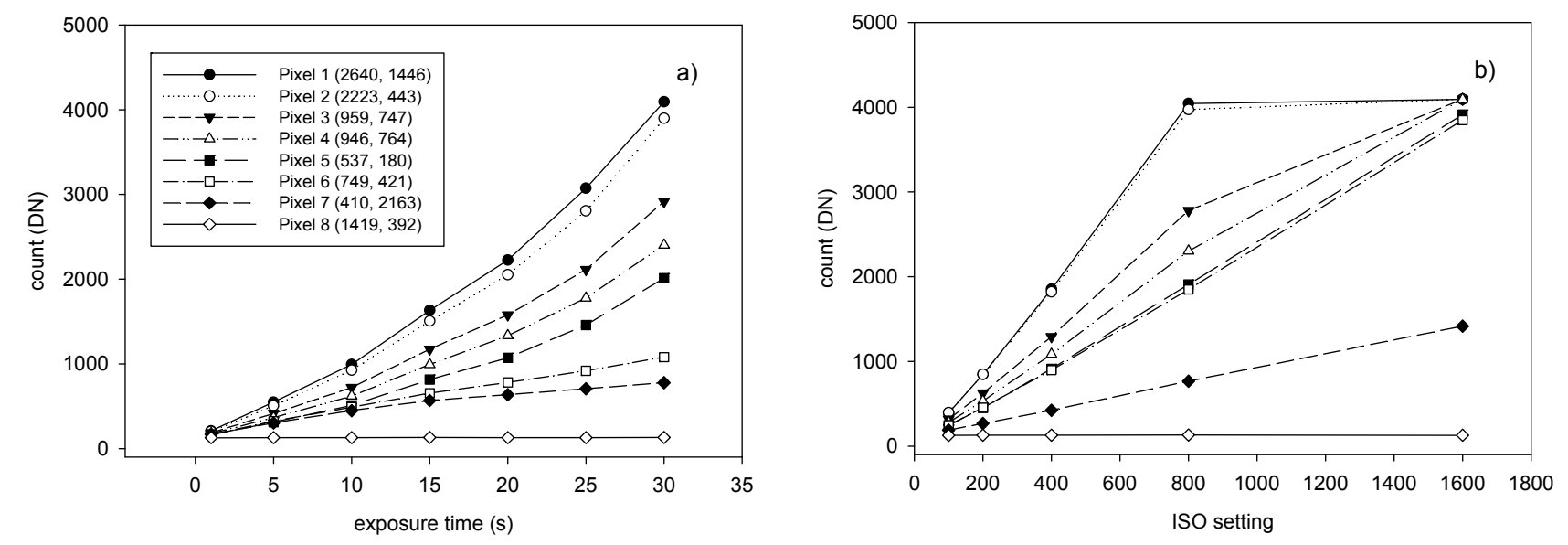

Fig. 5. Dependence of dark current for eight Canon 20Da pixels. Panel a): dependence on exposure time. Taken at $24^{\circ} \mathrm{C}$ with an ISO $=800$. Panel b): dependence on ISO setting. Taken at $24^{\circ} \mathrm{C}$ with an exposure time of $30 \mathrm{~s}$.

\subsection{Nikon D70}

The Nikon D70 has a 23.7 x 15.6 mm CCD sensor, with 6.1 million effective pixels in a grid of 3038 by 2014 pixels.

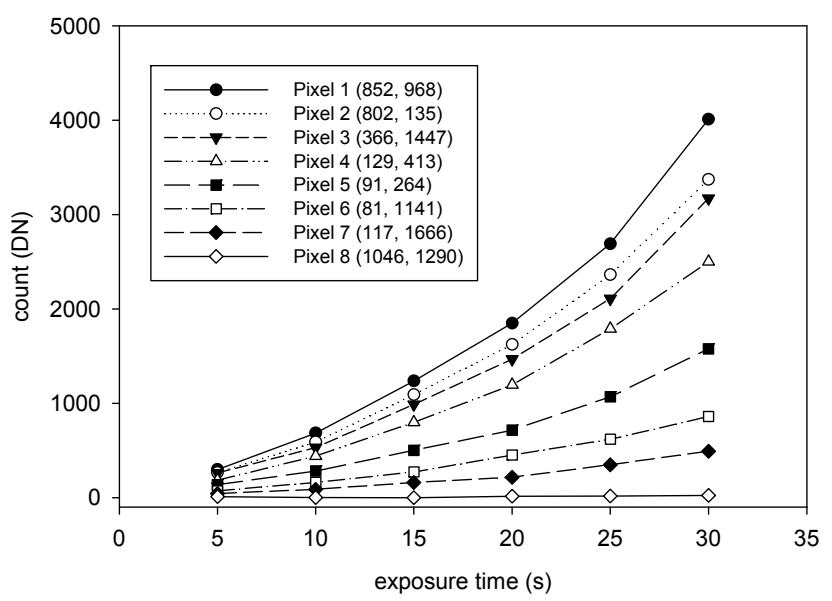

Fig. 6. Dependence of dark current for eight Nikon D70 pixels on exposure time. Taken at $24^{\circ} \mathrm{C}$ and ISO $=800$. 
Due to the added complications of taking frames to obtain accurate dark current data with the Nikon D70, only three images were taken at exposure times in ascending order of 5, 10, 15, 20, 25, and $30 \mathrm{~s}$. A median value was calculated from the three frames for each exposure time. Values for eight pixels are shown in Fig. 6. Pixels 1 to 7 show a sizeable increase in counts for increasing exposure time. They also demonstrate predictable behavior and therefore could be used as indicators of dark current for the entire imager. Pixel 8 lies close to zero for all exposure time values and is an example of typical pixels on the imager. In total the D70 has approximately 6000 pixels with dark noise exceeding 50 counts above the bias after $30 \mathrm{~s}$ of exposure time. These pixels are still numerous and roughly represent the portion of pixels on the imager that could be accurately improved by correction.

\subsection{Nikon D700}

The Nikon D700 has a $36 \times 23.9 \mathrm{~mm}$ CMOS sensor, with 12.1 million effective pixels in a grid of 4288 by 2845 pixels. The same number of images and exposure times were taken for the Nikon D700 as for the Nikon D70, and the median value of the images were calculated for each exposure time. Figure 7a shows the exposure time dependence of eight pixels on the imager. Pixels 1 to 7 show a large increase in counts with exposure time and also show a predictable behavior. Pixel 8 is an example of typical pixels on the chip and its values lie near zero for all exposure times. Additionally, three images were taken for ISO settings of $200,400,800$, and 1600 at $22^{\circ} \mathrm{C}$ and at an exposure time of 30s. A median value was taken for all ISO settings. Figure $7 \mathrm{~b}$ shows the dependence on ISO settings for the same pixels as shown in Fig. 7a. The same pixels that showed a large increase in dark counts due to increasing exposure time also show a large increase in counts due to increasing ISO setting. Thus, these pixels can accurately indicate the dark current in this imager for varying exposure times and ISO settings. Pixel 1, and to a lesser extent Pixel 2, begin to saturate over the range shown.
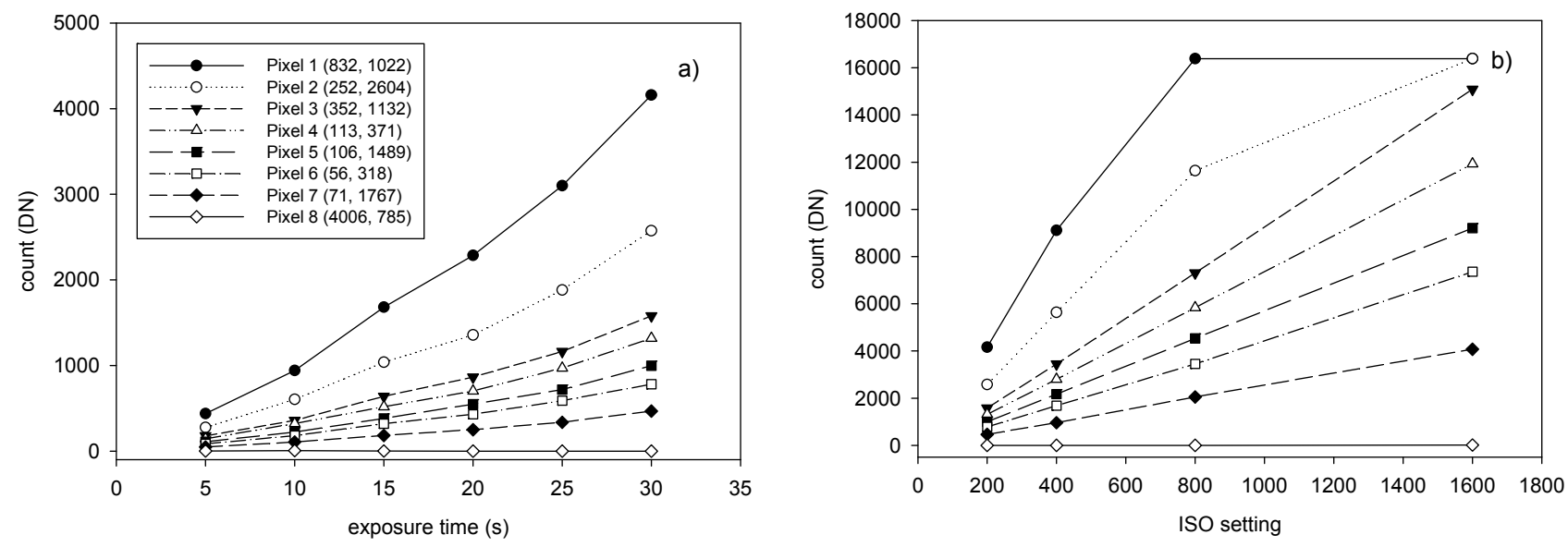

Fig. 7. Dependence of dark current for eight Nikon D700 pixels. Panel a): dependence on exposure time. Taken at $22^{\circ} \mathrm{C}$ and ISO $=200$. Panel b): dependence on ISO setting. Taken at $22^{\circ} \mathrm{C}$ with an exposure time of $30 \mathrm{~s}$.

\section{METHOD OF CORRECTION FOR DARK CURRENT}

Our method of correction is dependent upon finding the chip's hot pixels, pixels that have higher than average values of dark noise. These hot pixels are unique to every imager and arise due to impurities within the pixel. ${ }^{3}$ To find the hot pixels, a pixel is compared to its neighbors by using the following equation:

$$
\operatorname{hotpix}(x, y)=\sum_{i=-1}^{1} \sum_{j=-1}^{1} \operatorname{weight}(i, j) \cdot \operatorname{pixel}(x+i, y+j)
$$

where $(x, y)$ are the coordinates of the pixel on the image, and pixel $(x, y)$ is its dark count. The weight $(i, j)$ is chosen to be dependent on a neighbor's relative location to the pixel and so that the sum of all weighting factors equals zero. Different weighting factors and number of neighbors can be chosen. For the corrections done in this paper, the center pixel was weighted with a value of 1 , the pixels on the corners were weighted with a value of -0.05 , and the neighbors directly adjacent were weighted with a value of -0.2 . If all neighboring pixels have comparatively similar values to the center pixel, the value for hotpix $(x, y)$ will be close to zero. However, if all neighboring pixels have comparatively smaller values, the value for hotpix $(x, y)$ will be large. Next the values of hotpix $(x, y)$ are ranked and the $n$ hottest pixels 
are chosen. The average of hotpix $(x, y)$ for the $n$ hottest pixels is calculated and is used as a dark current indicator for the chip, $D_{\text {ind }}$. The values for hotpix $(x, y)$ are then fitted as a quadratic least squares function of the indicator such that:

$$
\operatorname{hotpix}(x, y)=a \cdot D_{\text {ind }}^{2}+b \cdot D_{\text {ind }}+c
$$

Pixels with poor fits are disregarded from the calculation. The values for the fitting parameters $a, b$, and $c$ can then be saved for the hottest pixels along with the coordinates of the hot pixels. The next step in the protocol is to determine the counts of all pixels with respect to $D_{\text {ind }}$. To accomplish this, the counts of each pixel for frames taken with different amounts of dark current, is fitted with a quadratic least squares fit versus the dark current indicator. Since the fit is later used to calculate the dark count, independently from the neighboring pixels, the actual count of the pixels (not the counts with respect to the neighboring pixels) is used to determine the fitting parameters. These parameters can then be used to evaluate the relative amount of dark current in any image within the calibrated regime.

It was shown that each imager had pixels that demonstrated predictable behavior of pixels on each imager to exposure time, gain, and temperature. These pixels could therefore be used to accurately predict the amount of dark current for all pixels on the imagers.

\section{IMAGE CORRECTION}

\subsection{Canon EOS-20D}

To determine the success of correction by the built-in noise reduction mode on the Canon 20D three frames were taken. The first frame was taken such that the noise reduction mode was turned on and the second and third frame were taken with noise reduction turned to off. The third image was subtracted from the second to obtain a corrected frame. As a measure for the success of correction of each method, the standard deviation is taken for the corrected frame. Ideally, the subtraction of two dark frames would result in a histogram with a narrow distribution that has values deviating from zero only due to random noise in the dark frames. Thus, a smaller standard deviation for the corrected frame's histogram indicates a better correction. However, a relatively small number of pixels with significantly smaller or larger values than zero after such a correction would not significantly alter the standard deviation, but would still show up as noticeable noise in an actual image. These outliers should be considered; nevertheless the standard deviation gives an easily comparable value that can be used to compare different methods of correction and is an indicator of the general quality of correction. The histogram of the frame with the Canon 20D's noise reduction mode turned on, shown in Fig. $8 \mathrm{a}$, has a standard deviation of 6.07 counts. The histogram is centered around a positive value likely due to a shift in offset such that the would-be negative values lie above zero as an actual image cannot contain negative values. The histogram of the corrected frame using another dark frame for correction, shown in Fig. 8b, has a standard deviation of 6.05 counts. The similar values of the standard deviations suggest that the Canon 20D does indeed take a second dark frame to subtract off the original image. Regardless, the Canon 20D's method of correction is shown to be no better than using an individual dark frame for subtraction as a method of correction.
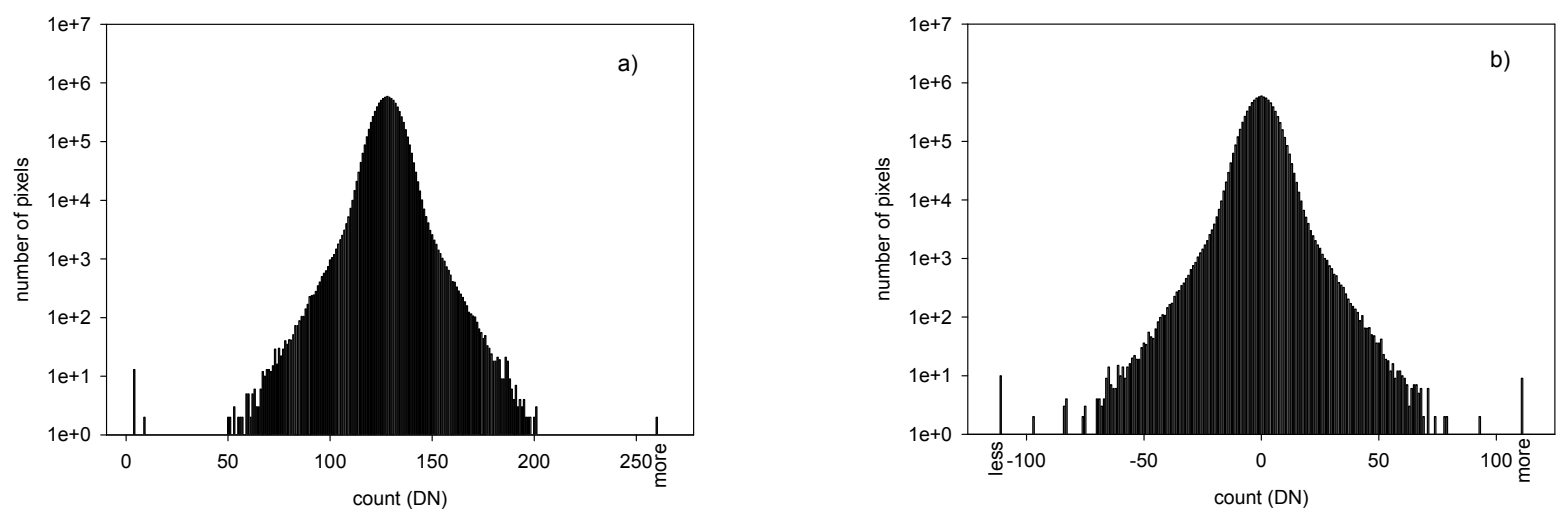

Fig. 8. Correction of a $30 \mathrm{~s}, 800$ ISO setting, $25^{\circ} \mathrm{C}$ image. Panel a): histogram of the frame taken with Canon 20D's Noise Reduction mode turned on. Panel b): histogram of a corrected frame using another individual image for correction.

To test the possibility of using the method of correction discussed at the beginning of this section on the Canon 20D, a series of 30 images, 5 each at exposure times of $5,10,15,20,25$, and $30 \mathrm{~s}$, were used to generate the coefficients of 

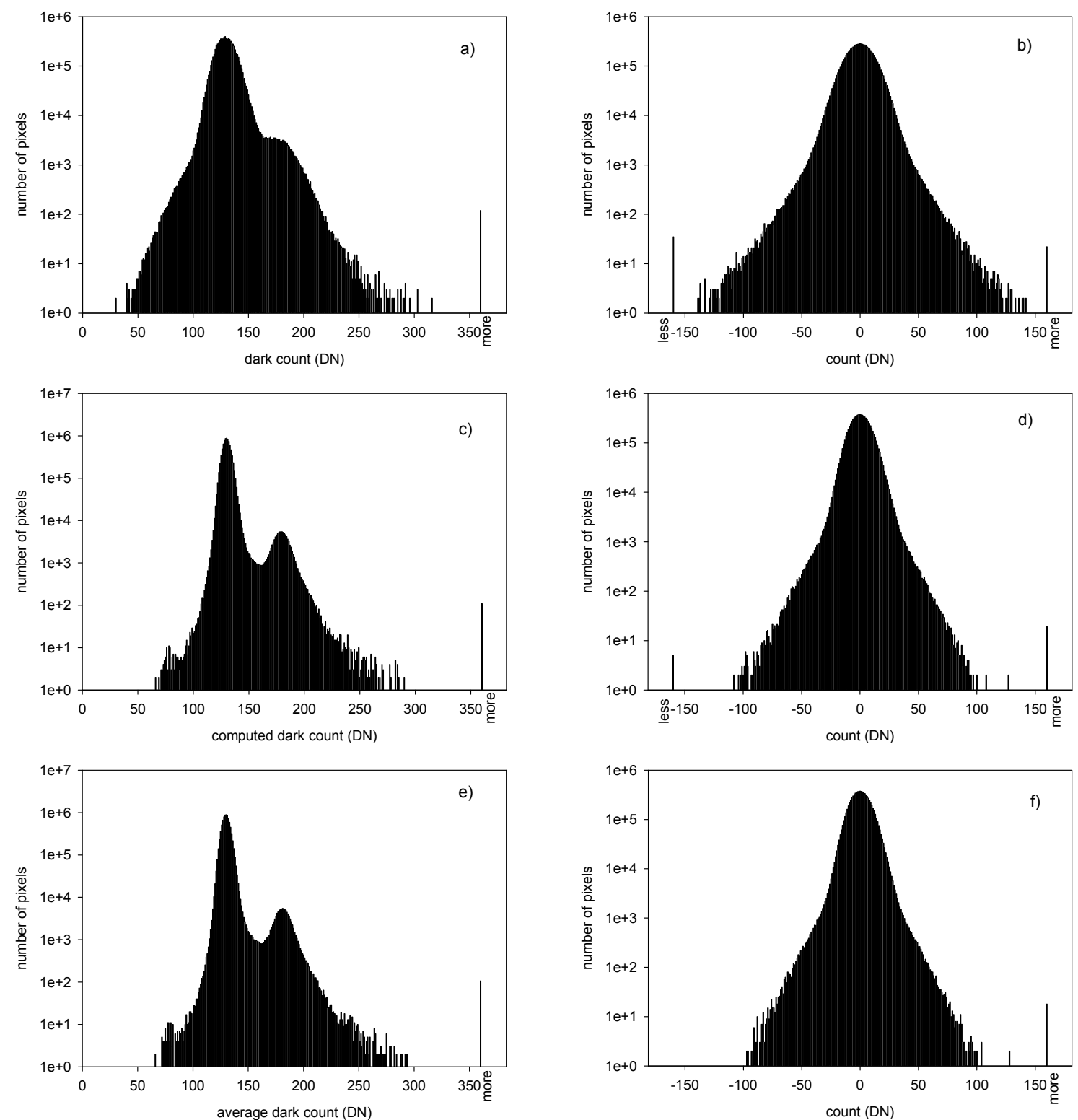

Fig. 9. Correction of a $30 \mathrm{~s}, \mathrm{ISO}=1600,23^{\circ} \mathrm{C}$ image. Panel a): histogram of the uncorrected frame. Panel b): histogram of the corrected frame using an individual frame for correction. Panel c): histogram of the constructed frame. Panel d): histogram of the corrected frame using the constructed frame for correction. Panel e): histogram of the master frame. Panel f): histogram of the corrected frame using the averaged frame for correction.

correction. This represents a total of $525 \mathrm{~s}$ of camera time. For comparison, we also used two other methods of correction, an averaged master frame and an individual dark frame. An averaged master frame was generated by taking the pixel by pixel average, with maximum and minimum values removed from the average, of nine $30 \mathrm{~s}$ frames. These images were taken after the image to be corrected and represent $270 \mathrm{~s}$ of camera time. Figure $9 \mathrm{a}$ shows the histogram of the uncorrected frame. Two peaks are apparent in the uncorrected frame, but are seen more distinctly in Fig. 9c, the histogram of the calculated frame, and Fig. 9e, the histogram of the master frame. The peaks can be seen more distinctly in the average and calculated frame due to longer observations and the corresponding reduction in random noise. These peaks are thought to be caused by specific types of impurities within the imager. ${ }^{7}$ The dark current in the uncorrected frame was removed by subtracting the frame generated by the three methods discussed. The original uncorrected image has a standard deviation of 15.36 counts. In Fig. 9b, the histogram for the correction using another individual frame is 
shown. This histogram has a standard deviation of 12.44 counts. Figure $9 \mathrm{~d}$ shows the histogram for the correction using the calculated frame that has a standard deviation of 9.31 counts. Figure $9 \mathrm{f}$ shows the corrected frame with a standard deviation of 9.28 counts. Thus the correction using the calculated frame has a $39 \%$ decrease in standard deviation compared to the original frame, shows a similar improvement as an averaged frame, and improves upon the correction using an individual frame.

Once the coefficients are calculated for an imager, dark frames can be quickly calculated for any exposure time within the calibrated region. Figure 10 shows the histograms related to the correction of a $15 \mathrm{~s}$ frame taken with an ISO setting of 1600 , and at a temperature of $23^{\circ} \mathrm{C}$. The histogram for the original uncorrected image is shown in Fig. 10a, and has a standard deviation of 11.59 counts. Figure 10c, shows the histogram for the calculated subtraction frame. The same coefficients that were calculated for the $30 \mathrm{~s}$ image were used to calculate this correction frame. Figure $10 \mathrm{~b}$ shows the histogram for the correction using an individual frame and has a standard deviation of 9.56 counts. Figure 10d shows the histogram for the correction using the calculated frame and has a standard deviation of 7.05 counts. The calculated frame decreases the standard deviation of the original image by 39\% and again improves upon the correction using a single image. Similar corrections were done using calculated frames for other exposure times within the calibrated region and improvements were seen over the entire region compared to the original frame and single frame correction.
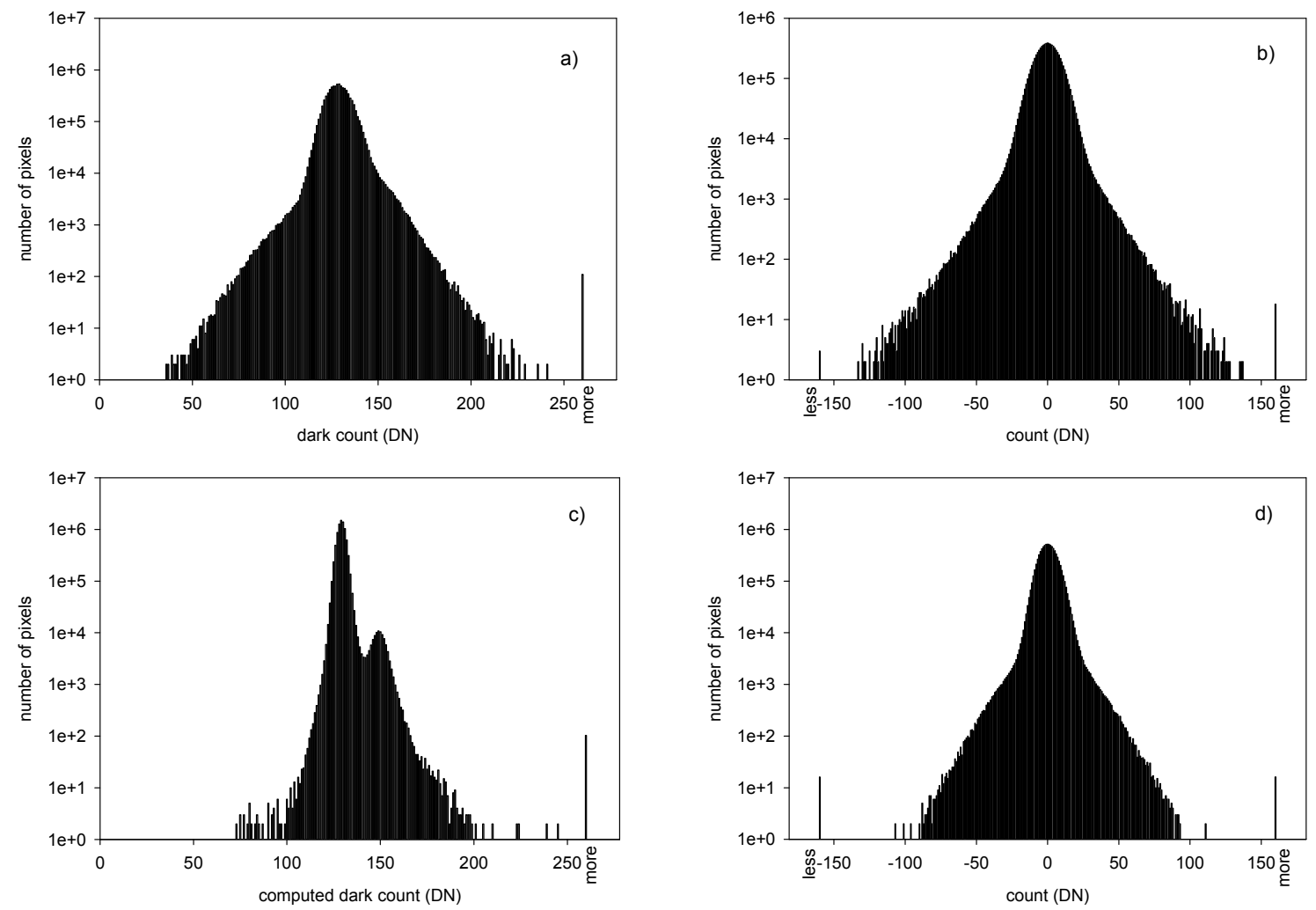

Fig. 10. Correction of a $15 \mathrm{~s}, \mathrm{ISO}=1600,23^{\circ} \mathrm{C}$ image. Panel a): histogram of the uncorrected frame. Panel b): histogram of the corrected frame using an individual frame for correction. Panel c): histogram of the constructed frame. Panel d): histogram of the corrected frame using the constructed frame for correction.

It remains to be shown that a series of dark frames taken at different temperatures can accurately predict and correct for dark current in an image taken at a given temperature and exposure time within the calibrated range. Images were taken at external temperatures of $24,30,35,40$, and $45^{\circ} \mathrm{C}$. Five frames at exposure times of $5,10,15,20,25$, and $30 \mathrm{~s}$, at each temperature were used to generate the coefficients of correction. This represents a total of 150 images and $2625 \mathrm{~s}$ of camera time. A calculated frame was generated for a dark frame taken for $20 \mathrm{~s}$, at $30^{\circ} \mathrm{C}$ with an ISO setting of 800 . The original uncorrected dark frame has a histogram with a standard deviation of 10.09 counts. For comparison an individual frame, taken under the same conditions as the original image, was used for correction and the resulting corrected frame's histogram is shown in Fig. 11a. This resulting histogram has a 
standard deviation of 7.09 counts. Figure 11b, shows the corrected frame's histogram using the calculated frame for correction. This histogram has a standard deviation of 5.14 counts, an improvement over using an individual frame, and $49 \%$ smaller than the standard deviation of the original frame.
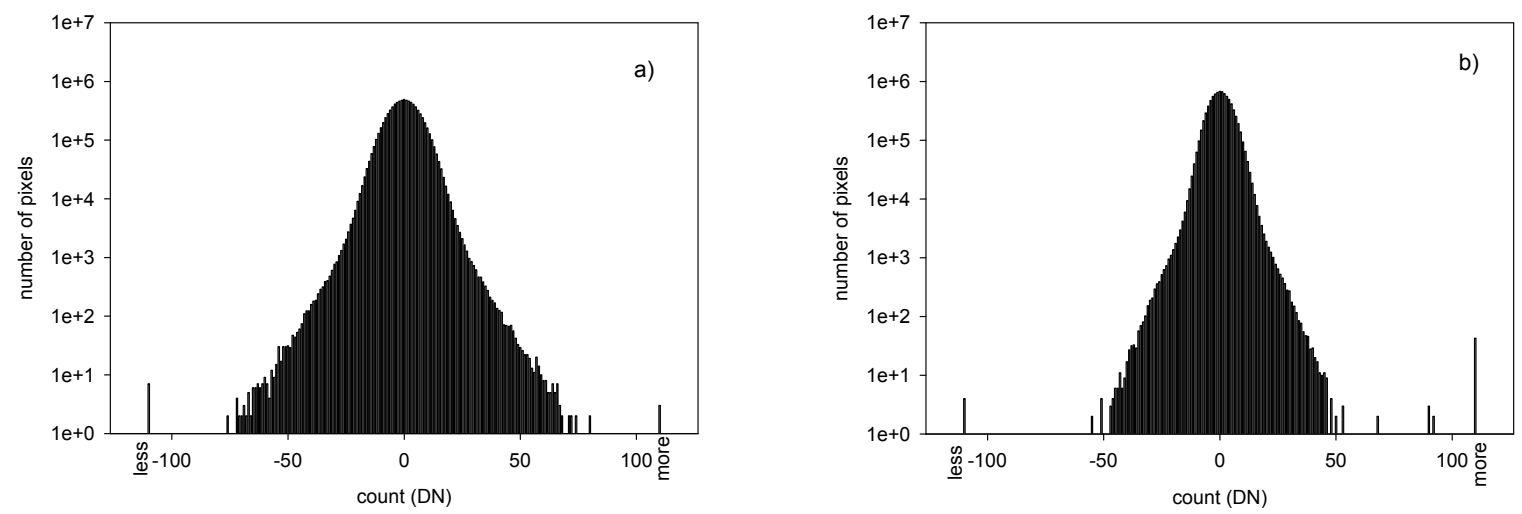

Fig. 11. Correction of a $20 \mathrm{~s}, \mathrm{ISO}=800,30^{\circ} \mathrm{C}$ image. Panel a: histogram of the corrected frame using another individual frame. Panel $b$ : histogram of the corrected frame using the computed frame.

The generation of averaged master frames allows for more accurate correction than a correction involving an individual frame as demonstrated above. However, the master frame requires significantly more camera time than an individual frame and is only accurate if the images composing the master frame are taken under the identical conditions as the image to be corrected. Figure 12a shows a histogram for the correction of the one hundredth image taken in the series of images used to generate Figure 3, using an average master frame generated from the first ten images in the series, with the highest and lowest value for each pixel removed from the average. As noted previously, the external temperature within the enclosure increased from $24.0^{\circ} \mathrm{C}$ to $25.2^{\circ} \mathrm{C}$ during this run; however the temperature of the sensor likely increased by significantly more. The increase in temperature of the sensor from the first ten frames to the one hundredth results in the bulge seen on the positive side of the peak. Due to many pixels having more dark current in the one hundredth frame than the first ten, a subtraction of the master frame results in these pixels having a positive value after the correction. Improvement in the standard deviation of the histogram is still seen; before correction the histogram of the image has a standard deviation of 12.91 counts, and after correction the histogram has a standard deviation of 8.08 counts. Figure $12 \mathrm{~b}$ shows a histogram of a corrected frame using a calculated frame from the coefficients for the same fitting as the previous correction. Using this method, the corrected frame has a standard deviation of 6.55 counts, a decrease of $49 \%$ compared to the original frame and an improvement over the master frame correction. Small temperature fluctuations of $1^{\circ} \mathrm{C}$ would not be uncommon over a session of image taking and, as shown in Section 2, the internal temperature of the imager itself likely changes by more for a long run of images. Therefore, a master frame generated from dark frames taken before or after many other frames will not provide accurate correction. The computed frames show better results since the dark count is calculated based on the actual chip temperature.
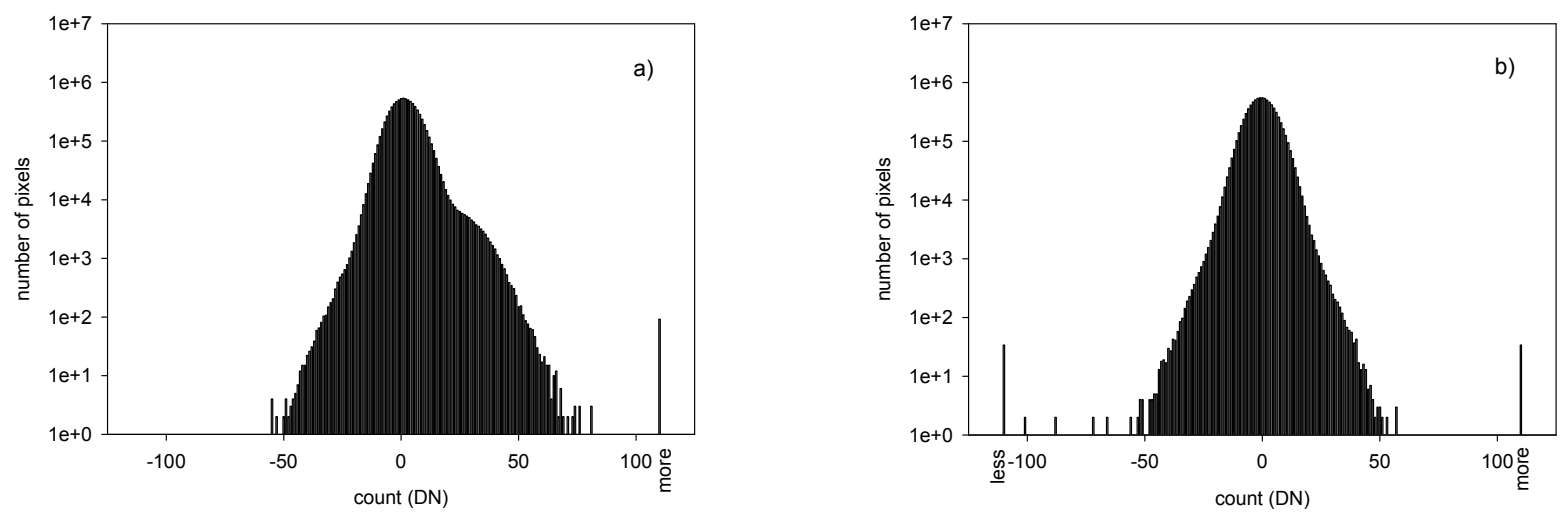

Fig. 12. Correction of a $30 \mathrm{~s}$, ISO $=800,25^{\circ} \mathrm{C}$ image. Panel a): histogram of the corrected frame using an averaged master frame taken at an external temperature of $24^{\circ} \mathrm{C}$. Panel b): histogram of the corrected frame using the computed frame. 


\subsection{Canon EOS-20Da}

In order to generate the coefficients for correction for the Canon 20Da, a series of 30 images, 5 each at exposure times of $5,10,15,20,25$, and $30 \mathrm{~s}$, were obtained, representing a total of $525 \mathrm{~s}$ of camera time. An uncorrected frame's histogram for a $25 \mathrm{~s}$ image with an $800 \mathrm{ISO}$ setting taken at $24^{\circ} \mathrm{C}$ is shown in Fig. 13a and has a standard deviation of 7.83 counts. Figure 13c shows the calculated frame's histogram. Figure 13b shows the histogram for the correction using another individual frame. This histogram has a standard deviation of 5.00 counts. Figure $13 \mathrm{~d}$ shows the histogram for the correction using the calculated frame. Its distribution is much narrower than the histogram in Fig. 13b and accordingly has a smaller standard deviation (3.65 counts). The correction using the calculated frame shows a $53 \%$ decrease in the histogram's standard deviation.
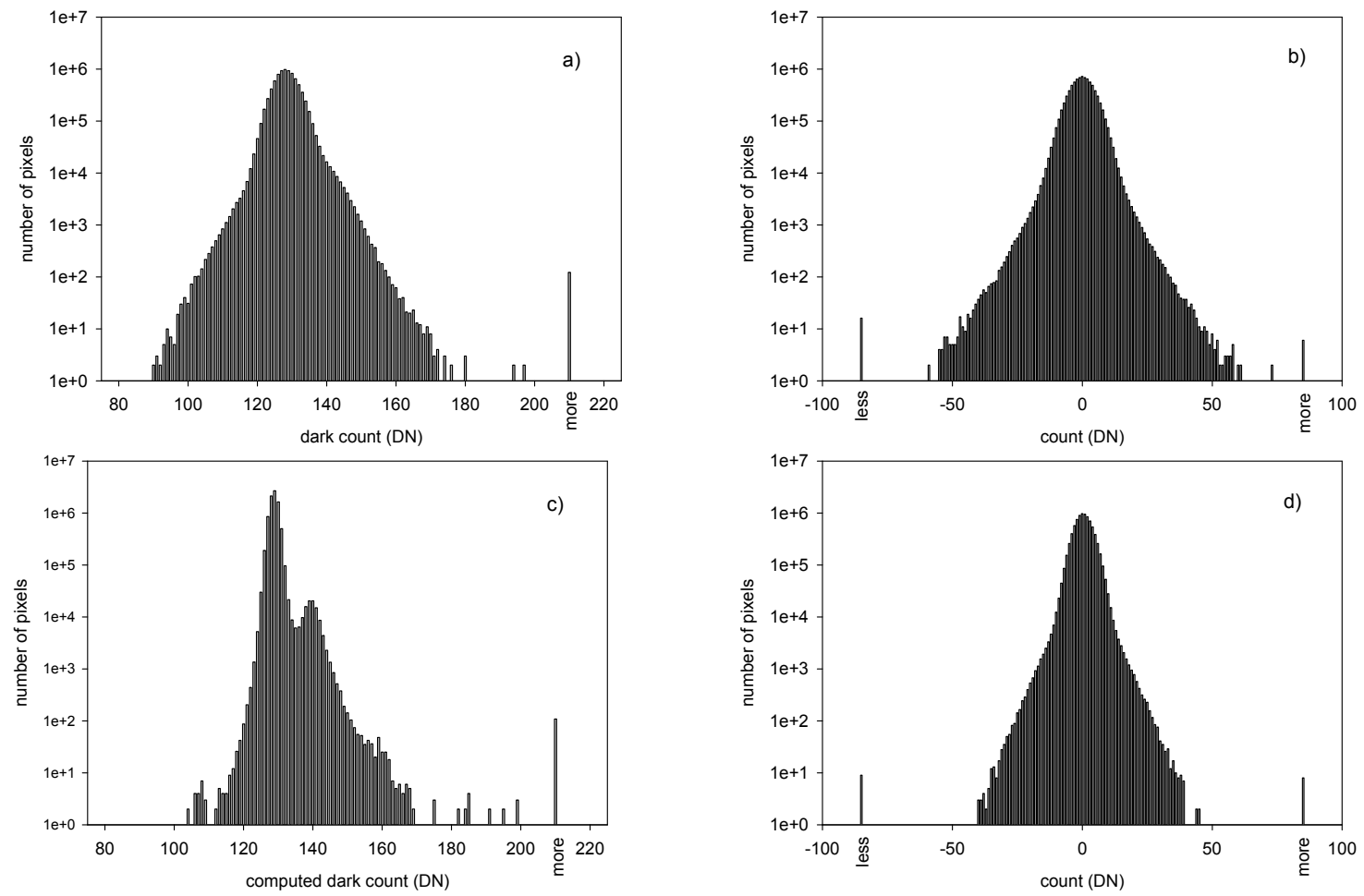

Fig. 13. Correction of a $25 \mathrm{~s}, \mathrm{ISO}=800,24^{\circ} \mathrm{C}$ image taken by the Canon $20 \mathrm{Da}$. Panel a): histogram of the uncorrected frame. Panel b): histogram of the corrected frame using an individual frame for correction. Panel c): histogram of the constructed frame. Panel d): histogram of the corrected frame using the constructed frame for correction.

\subsection{Nikon D70}

To calculate the coefficients for the Nikon D70, 18 images were used, 3 each for exposure times of 5, 10, 15, 20, 25, and $30 \mathrm{~s}$. This represents a total of $315 \mathrm{~s}$ of camera time. Figure 14a shows the histogram for the uncorrected frame with a standard deviation of 21.53 counts. Of note is the initial peak at 0 dark counts that is unusually large likely due to all would-be negative valued pixels being set to 0 . One also observes a smaller peak with significantly higher values at approximately 1600 counts. Figure $14 \mathrm{~b}$ shows the histogram of the corrected frame using another individual frame for correction. The resulting frame's histogram has a standard deviation of 11.80 counts. Figure $14 \mathrm{c}$ shows the histogram for the calculated frame. Figure $14 \mathrm{~d}$ shows the histogram of the corrected frame after subtraction of the calculated frame. The resulting histogram has a standard deviation of 8.58 counts, a $60 \%$ decrease compared to the original, and again showing an improvement in correction compared to using an individual frame. The distinctive shape of the resulting histogram is likely caused by the bias shifting in the original image. In addition to an improvement in standard deviation as compared to the individual image correction, the use of a computed frame results in fewer outliers with large counts after correction as is seen in Fig. 14b. The presence of these poorly corrected pixels using an individual frame for correction may be due to a shift in temperature of the sensor from the first image to the second and the corresponding change in dark counts from one image to the next for the hottest pixels. The change in dark counts of these pixels from one image to the next will degrade image quality. 

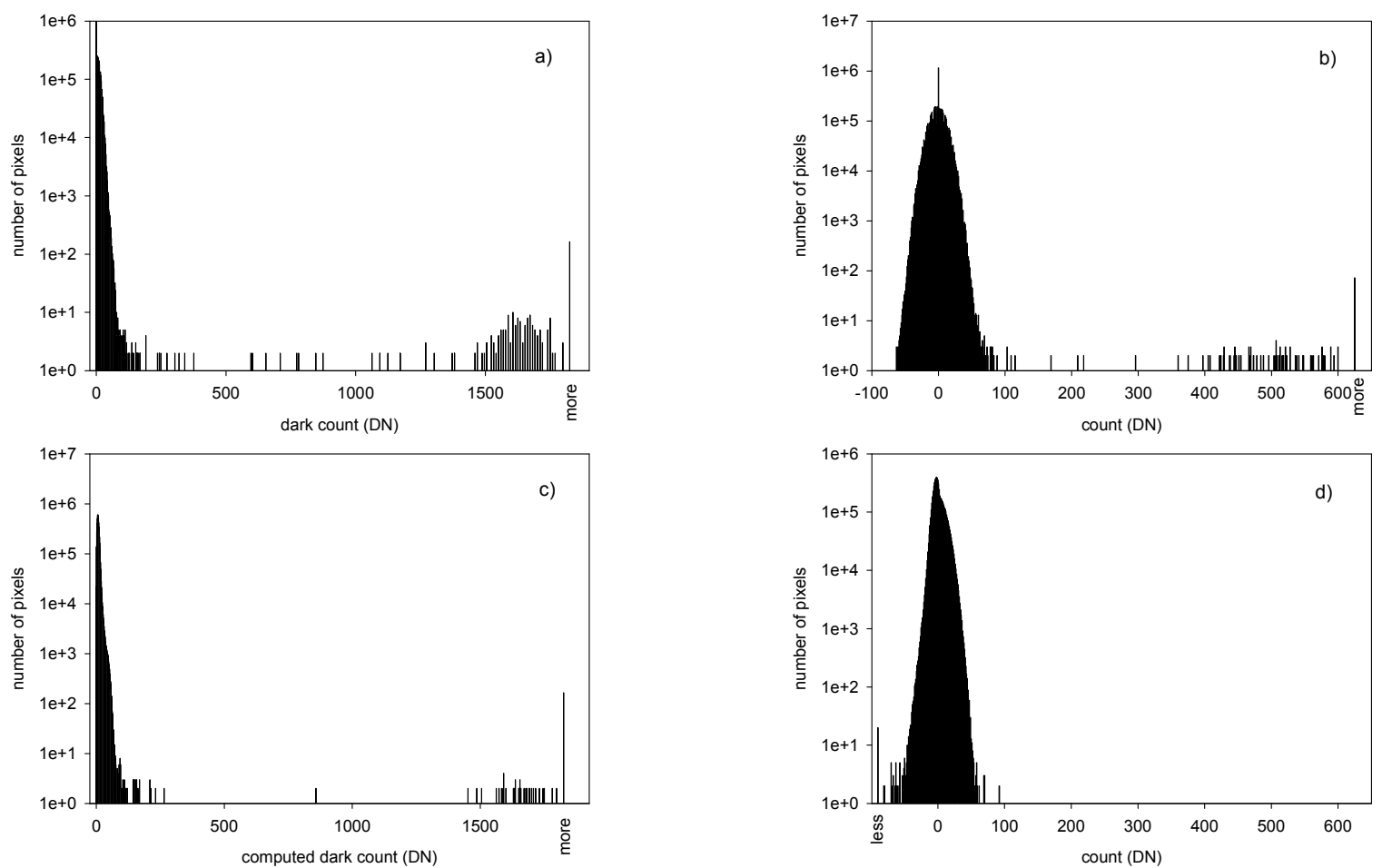

Fig. 14. Correction of a $25 \mathrm{~s}, \mathrm{ISO}=800,24^{\circ} \mathrm{C}$ image. Panel a): histogram of the uncorrected frame. Panel b): histogram of the corrected frame using another individual frame. Panel c): histogram of the calculated frame. Panel d): histogram of the corrected frame using the calculated frame.

\subsection{Nikon D700}
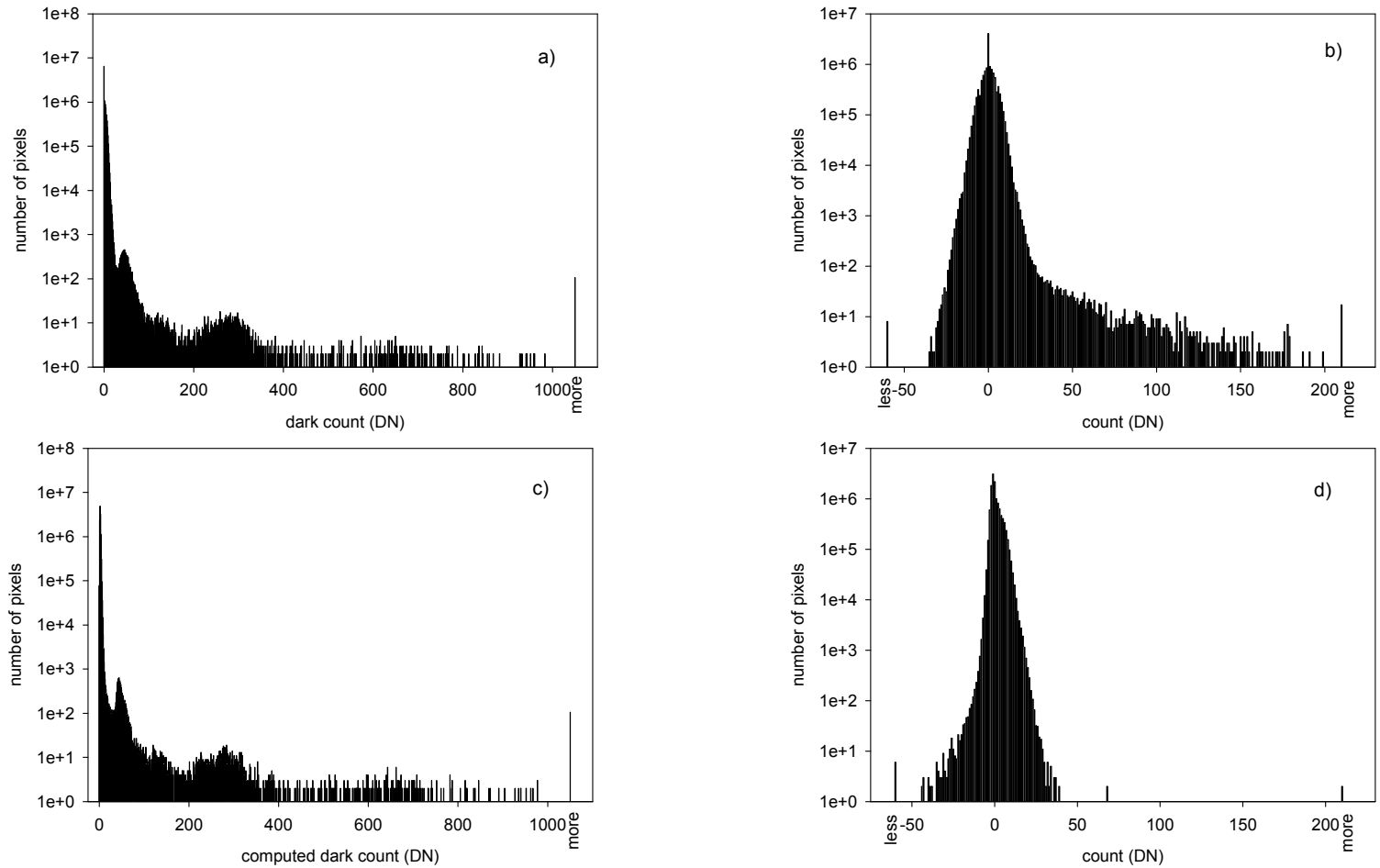

Fig. 15. Correction of a $25 \mathrm{~s}, \mathrm{ISO}=200,22^{\circ} \mathrm{C}$ image. Panel a): histogram of the uncorrected frame. Panel b): histogram of the corrected frame using another individual frame. Panel c): histogram of the calculated frame. Panel d): histogram of the corrected frame using the calculated frame. 
To calculate the coefficients for the Nikon D700, 18 images were used, 3 each for exposure times of 5, 10, 15, 20, 25, and $30 \mathrm{~s}$, a total of $315 \mathrm{~s}$ of camera time. As with the Nikon D70, due to the additional complications of taking frames with accurate dark current, an average master frame was not generated. Figure 15a shows the histogram for the uncorrected frame. The first two columns and the last column of the image have values consistently in the 700 count range that are not included in the histograms or the calculations. The initial peak begins at 0 counts and, similar to the Nikon D70, is unusually large due to the likely setting of all would-be negative valued pixels to 0 . In addition, there appears to be multiple peaks in the histogram at higher counts indicating the presence of different or multiple impurities. The uncorrected frame has a standard deviation of 7.95 counts. Figure $15 \mathrm{~b}$ shows the histogram of the corrected frame after using another individual frame for correction. The frame's histogram has a standard deviation of 4.00 counts. Figure $15 \mathrm{c}$ shows the histogram for the calculated frame. Figure $15 \mathrm{~d}$ shows the histogram of the corrected frame after subtraction of the calculated frame. The resulting histogram has a standard deviation of 2.92 counts, an improvement in correction using the calculated frame over the individual frame, and a $63 \%$ decrease in standard deviation from the original frame.

\section{SUMMARY}

We have shown that in DSLRs dark current behaves inconsistently under identical external conditions. This is likely due to temperature fluctuations within the camera itself. However, with proper care the imagers do generate predictable amounts of dark current and by using hot pixels as a dark current indicator, the dark current can be accurately corrected. The histograms of corrected frames using the method of calculating frames based on the hot pixels as indicators for dark current show a 40 to $60 \%$ decrease in the standard deviation compared to the original frame. Comparable results were shown between corrections using calculated frames and averaged master frames. Calculated frames have the advantages of requiring no additional frames to be taken after an initial calibration is done, and an image can be corrected retroactively using calculated frames without knowledge of the temperature when the image was taken. The method of using calculated frames consistently shows improvements over using a single frame for correction. Further, we have shown that camera manufactures' methods for removing dark current can be improved upon for many possible configurations of temperature, gain value, and exposure time.

\section{REFERENCES}

[1] Widenhorn, R., Rest, A., Blouke, M. M., Berry, R. L., and Bodegom, E., "Computation of dark frames in digital imagers," Proc. of SPIE 6501, 650103 (2007)

[2] S.M. Sze, "Physics of Semiconductor Devices," (John Wiley \& Sons, 1967)

[3] McGraph, R.D., Doty, J., Lupino, G., Ricker, G., and Vallerga, J., "Counting of deep-level traps using a chargecoupled device," IEEE Trans. Electron Devices, vol. ED-34, 2555, (1987)

[4] McColgin, W.C., Lavine, J.P., Kyan, J., Nichols, D.N., and Stancampiano, C.V., "Dark current quantization in CCD image sensors," International Electron Device Meeting 1992, p. 113, 13-16 (1992)

[5] Porter, W.C., Kopp, B., Dunlap, J.C., Widenhorn, R., and Bodegom, E., "Dark current measurements in a CMOS imager," Proc. of SPIE 6816, 68160C (2008)

[6] Buil, C., "Comparative test Canon 10D/ Nikon D70 in the field of deep-sky astronomy", http://astrosurf.com/buil/d70v10d/eval.htm

[7] McColgin, W.C., Lavine, J.P., and Stancampiano, C.V., "Probing metal defects in CCD image sensors," Proc. of Mat. Res. Soc. Symp. 378, 713 (1995) 